\title{
Extraction of Toxic Metabolites from Selected Strains of Aspergillus niger and Fusarium solani
}

\author{
Withanage S.V. ${ }^{1 *}$, Fernando T.H.P.S. ${ }^{2}$, Subasinghe S.M.C.U.P. ${ }^{1}$ \\ ${ }^{1}$ Department of Forestry and Environmental Science, University of Sri Jayewardenepura, Sri Lanka \\ ${ }^{2}$ Plant Pathology and Microbiology Department, Rubber Research Institute, Sri Lanka \\ *sohaniw@gmail.com
}

\begin{abstract}
Aspergillus niger and Fusarium solani have been reported to show the ability of inducing agarwood formation in Gyrinops walla. Both these species are also well known for the ability to produce mycotoxins; products from secondary metabolism of fungi, with varied biochemical structures and modes of action. Therefore it is worth knowing the characteristics of the toxins formed by these fungal species before using them as potential inoculants. Since the production of mycotoxin is limited to certain fungal strains, the present study aimed to identify the potential of selected strains of A. niger and F. solani for toxin production and its extraction.

Therefore, two strains of $A$. niger and two strains of $F$. solani were screened to identify their ability to produce mycotoxins in Yeast Extract Glucose (YEG) and CzapekDox (CDB) liquid media. These media were inoculated with three agar plugs from fully grown plate cultures and incubated at room temperature under normal light and dark regimes. The filtrates were obtained after incubation periods of 4, 8, 10, 12, 14, 16, 21 and 28 days. Production of toxins was identified by assessing the damage caused on the surface of Piper nigarum leaves by a known volume $(20 \mu \mathrm{l})$ of filtrate. Toxicity of culture filtrates were evaluated qualitatively by a graded scale of Group 1 to Group 5.

According to the results, both strains of A. niger produced positive results for the production of mycotoxin. However, Asp-MBL $1-\mathrm{C}$ strain showed the maximum toxin production in YEG medium after 4 days of incubation while Asp-U strain showed the maximum toxin production in the same medium after 12 days of incubation. The maximum toxin production in CDB medium was observed after 14 days of incubation. More intense leaf damage was observed to be associated with Asp-U strain in CDB medium. Out of two strains of $F$. solani tested in two selected media, only U-Fus strain produced positive results in YEG medium, where maximum toxicity was observed after 10 days of incubation.
\end{abstract}

Keywords: Mycotoxin, Aspergillus niger, Fusarium solani, agarwood inducement

Proceedings of the International Forestry and Environment Symposium 2016, Department of Forestry and Environmental Science, University of Sri Jayewardenepura, Sri Lanka. 